\title{
Gyeji-tang water extract exerts anti-inflammatory activity through inhibition of ERK and NF-KB pathways in lipopolysaccharide-stimulated RAW 264.7 cells
}

\author{
Sae-Rom Yoo ${ }^{1}$, Yeji Kim ${ }^{1,3}$, Mee-Young Lee ${ }^{1}$, Ohn-Soon Kim², Chang-Seob Seo ${ }^{1}$, Hyeun-Kyoo Shin ${ }^{1}$
} and Soo-Jin Jeong ${ }^{2,4^{*}}$

\begin{abstract}
Background: Gyeji-tang (GJT, Guizhi Tang in Chinese, Keishi-to in Japanese) is a traditional herbal decoction composed of 5 medicinal herbs. GJT has been used to treat the common cold, headaches, and fever in Asian countries including Korea, China, and Japan. In the present study, we investigated the inhibitory effect of a water extract of GJT on inflammatory response using the murine macrophage cell line, RAW 264.7.

Methods: RAW 264.7 macrophages were treated with lipopolysaccharide (LPS) to upregulate inflammatory genes. Cells were pretreated with various concentrations of GJT for $4 \mathrm{~h}$ and stimulated with LPS for an additional $20 \mathrm{~h}$. Productions of tumor necrosis factor-alpha (TNF-a), interleukin-6 (IL-6), cyclooxygenase-2 (COX-2), and prostaglandin $\mathrm{E}_{2}$ $\left(\mathrm{PGE}_{2}\right)$ were measured by enzyme-linked immunosorbent assays (ELISAs). Protein expressions of heme oxygenase (HO)1, extracellular signal-regulated kinase (ERK), and nuclear factor kappa-B (NF-KB) were analyzed by immunoblotting.

Results: Treatment with the GJT extract enhanced expression of HO-1 in macrophages without cytotoxicity. GJT extract significantly inhibited proinflammatory cytokines TNF-a and IL-6 in LPS-stimulated cells. GJT suppressed LPSinduced COX-2 expression, leading to a decrease in COX-2-derived PGE 2 level. In addition, GJT extract prevented phosphorylation of ERK and NF-KB translocalization to the nucleus in LPS-treated RAW 264.7 cells.
\end{abstract}

Conclusion: These data suggest that GJT has anti-inflammatory possibly through blocking ERK and NF-KB signaling pathways.

Keywords: Gyeji-tang, Supression of inflammation, Macrophage, Extracellular signal-regulated kinase, Nuclear factor kappa B

\section{Background}

Inflammation is a tissue protective response against potentially harmful stimuli such as bacteria, damaged cells, or irritants $[1,2]$. Chronic inflammation is associated with pathogenesis of the various diseases such as cancer, atherosclerosis, rheumatoid arthritis, and type 2 diabetes.

\footnotetext{
* Correspondence: sjijeong@kiom.re.kr

${ }^{2}$ KM Convergence Research Division, Korea Institute of Oriental Medicine,

1672 Yuseong-daero, Yuseong-gu, Daejeon 34054, Republic of Korea

${ }^{4}$ Korean Medicine Life Science, University of Science and Technology,

Daejeon, Republic of Korea

Full list of author information is available at the end of the article
}

Macrophages are significantly involved in the initiation and maintenance of the inflammatory process through secretion of inflammatory cytokines [3]. Blocking proinflammatory cytokines such as tumor necrosis factor-alpha (TNF- $\alpha$ ) or interleukin-6 (IL-6) is considered as an attractive therapeutic approach to inflammation $[4,5]$. In addition to inflammatory cytokines, prostaglandin $\mathrm{E} 2\left(\mathrm{PGE}_{2}\right)$ as a proinflammatory mediators plays an important role in inflammatory response. $\mathrm{PGE}_{2}$ is synthesized by cyclooxygenase-2 (COX-2) that is activated by proinflammatory stimuli such as cytokines, endotoxin, or growth factors [6]. These 
proinflammatory regulators can be controlled by several intracellular molecular pathways such as those involving mitogen-activated protein kinases (MAPKs) and nuclear factor kappa-B (NF-kB) [7]. Chronic inflammation and its related diseases are associated with the oxidative stress that generates proinflammatory mediators and cytokines. The antioxidative enzyme heme oxygenase 1 (HO-1) has anti-inflammatory properties and is therefore thought to be a potential molecular target for treating inflammatory diseases $[8,9]$. In macrophages, activation of HO-1 inhibits cytokine secretion $[10,11]$ and suppresses COX-2 expression [12].

Gyeji-tang (GJT, Guizhi Tang in Chinese, Keishi-to in Japanese), a traditional Korean medicine, has been used to treat cold, headache, and fever in Asian countries including Korea, China and Japan [13]. GJT consists five herbs. However, the scientific evidence to support antiinflammatory effect of GJT is rare. To date, several groups reported effects of GJT on pancreatic acinar cell injury [14], diabetes mellitus [15], and Guizhi decoction syndrome [16]. In the present study, we investigated anti-inflammatory mechanisms of GJT water extract using lipopolysaccharide (LPS)-stimulated RAW 264.7 murine macrophages. Inhibitory effects of GJT against the inflammatory response was elucidated by measuring production of TNF- $\alpha$, IL- 6 and $\mathrm{PGE}_{2}$, and analyzing MAPK and NF-KB pathways, and $\mathrm{HO}-1$ expression.

\section{Methods}

\section{Plant materials}

The 5 medicinal herbs comprising GJT were purchased from Kwang Myung Dang Medicinal Herbs (Ulsan, Korea) as shown in Table 1 . The taxonomic authenticity of these medicinal herbs was confirmed by Prof. Je Hyun Lee, Dongguk University, Gyeongju, Korea. Voucher specimen (2012-KE46-1 through KE46-5) have been deposited at the K-herb Research Center, Korea Institute of Oriental Medicine.

\section{Preparation of GJT water extract}

Five medicinal herbs was mixed as shown in Table 1 (total $5.0 \mathrm{~kg} ; 30.0 \mathrm{~g} \times 166.7)$ and extracted in a 10-fold mass of water at $100{ }^{\circ} \mathrm{C}$ for $2 \mathrm{~h}$ under pressure $\left(1 \mathrm{kgf} / \mathrm{cm}^{2}\right)$ using an electric extractor (COSMOS-660; Kyung Seo Machine Co., Incheon, Korea). The water extract was then filtered through a standard sieve (No. 270, $53 \mu \mathrm{m}$; Chung Gye Sang Gong Sa, Seoul, Korea). The solution was freezingdrying to give a powder using PVT100 freeze dryer (IlShinBioBase, Yangju, Korea). The yield of the GJT water extract was $9.75 \%$ (487.5 g).

\section{Cell culture}

The murine macrophage cell line, RAW 264.7, was obtained from the American Type Culture Collection (Rockville, MD). The cells were cultured in Dulbecco's modified Eagle's medium (Gibco Inc., Grand Island, NY) supplemented with $5.5 \%$ heat-inactivated fetal bovine serum (Gibco Inc.), penicillin (100 U/mL), and streptomycin $(100 \mu \mathrm{g} / \mathrm{mL})$ under an atmosphere of $5 \% \mathrm{CO}_{2}$ in an incubator at $37^{\circ} \mathrm{C}$.

\section{Cytotoxicity assay}

Cell viability assay was performed to determine the cytotoxicity of GJT using a Cell Counting Kit-8 (CCK-8; Dojindo, Kumamoto, Japan). Cells were plated into a 96well microplates at $3 \times 10^{3}$ cells/well and treated with various concentrations of GJT for $24 \mathrm{~h}$. After incubation with CCK-8 reagent for $4 \mathrm{~h}$, optical density (OD) at $450 \mathrm{~nm}$ was measured by using a Benchmark plus microplate reader (Bio-Rad Laboratories, Hercules, CA). Cell viability was calculated using the following equation:

$$
\text { Cell viability }(\%)=\frac{\text { Mean OD in GJT treated cells }}{\text { Mean OD in untreated cells }} \times 100
$$

\section{ELISAs for TNF- $a$, IL- 6 , and PGE}

Cells were pretreated with various concentrations of GJT for $4 \mathrm{~h}$ and stimulated with LPS $(1 \mu \mathrm{g} / \mathrm{mL})$ for an additional $20 \mathrm{~h}$. Production of TNF- $\alpha$, IL-6, and PGE 2 in the culture supernatants was measured using commercial ELISA kits from R\&D systems (Minneapolis, MN), BD Biosciences (Mountain View, CA), and Cayman Chemical Co. (Ann Arbor, MI), respectively.

Table 1 Herbal composition of GJT

\begin{tabular}{llll}
\hline Herbal medicine & Scientific name & Origin & Amount (g) \\
\hline Cinnamomi Ramulus & Cinnamomum cassia & Vietnam & 11.25 \\
Paeoniae Radix & Paeonia lactiflora & Uiseong, Korea & 7.50 \\
Glycyrrhizae Radix et Rhizoma & Glycyrrhiza uralensis & China & 3.75 \\
Zingiberis Rhizoma Crudus & Zingiber officinale & Yeongcheon, Korea & 3.75 \\
Zizyphi Fructus & Zizyphus jujuba & Yeongcheon, Korea & 3.75 \\
Total amount & & & 30.00 \\
\hline
\end{tabular}


Reverse transcription-polymerase chain reaction (RT-PCR) Total RNA was extracted using Trizol reagent (Invitrogen Life Sciences, Carlsbad, CA, USA) according to the manufacturer's instructions. cDNA was synthesized from $1 \mu \mathrm{g}$ of total RNA using an iScript cDNA synthesis kit (Bio-Rad Laboratories, Hercules, CA, USA) and subjected to PCR reactions with rTaq DNA polymerase (ELPIS Biotech Inc., Daejeon, South Korea). The relative expression of COX-2 was analyzed using $\beta$-actin as an internal control. The primer sequence for $C O X-1$ was forward 5'-AGG AGA TGG CTG CTG AGT TGG-3' and reverse $5^{\prime}$-AAT CTG ACT TTC TGA GTT GCC3', COX-2 was forward 5'-GTA TCA GAA CCG CAT TGC CTC TGA-3' and reverse 5'-CGG CTT CCA GTA TTG AGG AGA ACA GAT-3', and $\beta$-actin was forward 5' -ACC GTG AAA AGA TGA CCC AG-3' and reverse 5 '-TAC GGA TGA CAA CGT CAC AC-3'. The PCR conditions were 25 cycles of $94{ }^{\circ} \mathrm{C}$ for $30 \mathrm{~s}, 57{ }^{\circ} \mathrm{C}$ ( $\beta$-actin) or $59{ }^{\circ} \mathrm{C}$ for $1 \mathrm{~min}$, and $72{ }^{\circ} \mathrm{C}$ for $1.5 \mathrm{~min}$. The amplification products were then separated by electrophoresis on $1 \%$ agarose gels and detected using a Molecular Imager Gel Doc XR System (Bio-Rad Laboratories, Hercules, CA, USA).

\section{Western blotting}

Whole cell extract was prepared by suspending cells in an extraction lysis buffer (Sigma-Aldrich, St. Louis, MO) containing protease inhibitor cocktail (Roche Applied Science, Indianapolis, IN). Nuclear extract was isolated using NE-PER Nuclear and Cytoplasmic Extraction reagents (Thermo Scientific, Rockford, IL) according to the manufacturer's protocol. Protein concentrations in the extracts were determined using a Bio-Rad Protein Assay reagent (Bio-Rad, Hercules, CA). Equal amount of cell extract proteins $(30 \mu \mathrm{g})$ were resolved by $4 \%-20 \%$ sodium dodecyl sulfate-polyacrylamide gel electrophoresis (SDSPAGE) and transferred to a polyvinylidene fluoridemembranes. The membrane was incubated with blocking solution (5\% skim milk in Tris-buffered saline containing Tween 20 (TBST), followed by an overnight incubation at $4{ }^{\circ} \mathrm{C}$ with the appropriate primary antibodies; anti-phospho-p38 MAPK, anti-phospho-ERK, anti-phospho-JNK (Cell Signaling, Danvers, MA), HO-1 (Abcam, Boston, MA), NF-кB p65, HO-1, and $\beta$-actin (Santa Cruz Biotechnology, Dallas, TX). The membranes were washed three times with TBST, and then incubated with a horseradish peroxidase (HRP)-conjugated secondary antibody (Jackson ImmunoResearch, West Grove, PA) for $1 \mathrm{~h}$ at room temperature. The membranes were washed three times with TBST again, and then developed using an enhanced chemiluminescence kit (Thermo Scientific). Image capture was performed using a Chemi-Doc XRS system (Bio-Rad).

\section{Immunofluorescence staining}

Cells were plated onto poly-l-lysine coated glass slides and fixed in $4 \%(\mathrm{v} / \mathrm{v})$ methanol free formaldehyde solution $(\mathrm{pH} 7.4)$ at $4{ }^{\circ} \mathrm{C}$ for $25 \mathrm{~min}$. The cells were permeabilized in $0.2 \%(\mathrm{w} / \mathrm{v})$ Triton X-100, blocked in $5 \%(\mathrm{w} / \mathrm{v})$ bovine serum albumin (BSA) in humidified chamber, followed by immunostaining with NF- $\mathrm{B}$ p 65 antibody (Santa Cruz Biotechnology, Santa Cruz, CA) and Texas Red-conjugated secondary antibody. The cells were mounted with mounting medium with coverslips with a mounting medium containing DAPI (Vector Laboratories, Inc, Burlingame, CA) and visualized under an FlouviewFV10i confocal microscope (Olympus, Tokyo, Japan).

\section{Chemicals}

The reference standards, gallic acid, benzoic acid, and coumarin were purchased from Sigma-Aldrich (St. Louis, MO, USA). Albiflorin, paeoniflorin, liquiritin, cinnamic acid, cinnamaldehyde, glycyrrhizin, and 6-gingerol were purchased from Wako (Osaka, Japan). Benzoylpaeoniflorin and liquiritin apioside were purchased from Biopurify Phytochemicals (Chengdu, China) and Shanghai Sunny Biotech (Shanghai, China). A standard stock solution of these components were dissolved in methanol at concentrations of $1.0 \mathrm{mg} / \mathrm{mL}$. For HPLC analysis of the GJT extract, $100 \mathrm{mg}$ of lyophilized GJT extract was dissolved in $20 \mathrm{~mL}$ of distilled water and then the solution was diluted to 10-fold for quantitative analysis of paeoniflorin. Solutions were filtered through a SmartPor GHP $0.2 \mu \mathrm{m}$ syringe filter (Woong Ki Science Co., Seoul, Korea) before application to the column.

\section{HPLC analysis of GJT}

Quantitative analysis of 12 compounds present in the GJT extract was performed using a Shimadzu LC-20A HPLC system (Shimadzu Co., Kyoto, Japan) consisting of a solvent delivery unit, an on-line degasser, a column oven, an autosampler, and a PDA detector. The data were acquired and processed by LabSolution software (version 5.54 SP3l Shimadzu Co.). The analytical column used was a SunFire $C_{18}(250 \times 4.6 \mathrm{~mm}$; particle size $5 \mu \mathrm{m}$, Waters, Milford, MA, USA) and was maintained at $40{ }^{\circ} \mathrm{C}$. The mobile phases consisted of water (A) and acetonitrile (B), which were both containing $0.1 \%(\mathrm{v} / \mathrm{v})$ formic acid. The gradient flow was as follows: $5 \%-$ $60 \%$ B for $0-30 \mathrm{~min}, 60 \%-100 \%$ B for $30-40 \mathrm{~min}$, $100 \%$ B for $40-45 \mathrm{~min}$, and $100-5 \%$ B for $45-50 \mathrm{~min}$. The flow-rate was $1.0 \mathrm{~mL} / \mathrm{min}$ and injection volume was $10 \mu \mathrm{L}$.

\section{Statistical analysis}

The data are expressed as the mean \pm SEM. Data were analyzed using one-way analysis of variance and Dunnett's 
multiple comparisons test. $P<0.05$ was considered significant.

\section{Results}

GJT enhances HO-1 expression in RAW264.7 cells

Macrophages play an important role in the process of inflammation including its initiation, maintenance, and resolution through the production of pro- or antiinflammatory cytokines [3]. In the present study, RAW 264.7 murine macrophages were used to investigate anti-inflammatory effects of the GJT extract. To evaluate the cytotoxicity of GJT against RAW 264.7 cells, we performed a CCK assay. Cells were treated with various concentrations of GJT extract (15.63, 31.25, 62.50, $125.00,250.00,500.00$, or $1000.00 \mu \mathrm{g} / \mathrm{mL}$ ) for $24 \mathrm{~h}$. As shown in Fig. 1a, the cell viability was maintained at $95.38 \pm 0.87 \%$ at $\leq 250 \mu \mathrm{g} / \mathrm{mL}$. However, higher concentrations of GJT extract (500 and $1000 \mu \mathrm{g} / \mathrm{mL}$ ) decreased cell viability by $74.28 \pm 0.74 \%$ and $59.89 \pm 1.05 \%$, respectively. Subsequent assays in RAW 264.7 cells were conducted in nontoxic concentrations of GJT extract. To investigate the inhibitory effects of GJT extract on inflammation, we initially analyzed $\mathrm{HO}-1$ expression in RAW 264.7 cells. Treatment with GJT extract induced HO-1 expression in a dose-dependent manner at noncytotoxic concentrations (Fig. 1b).

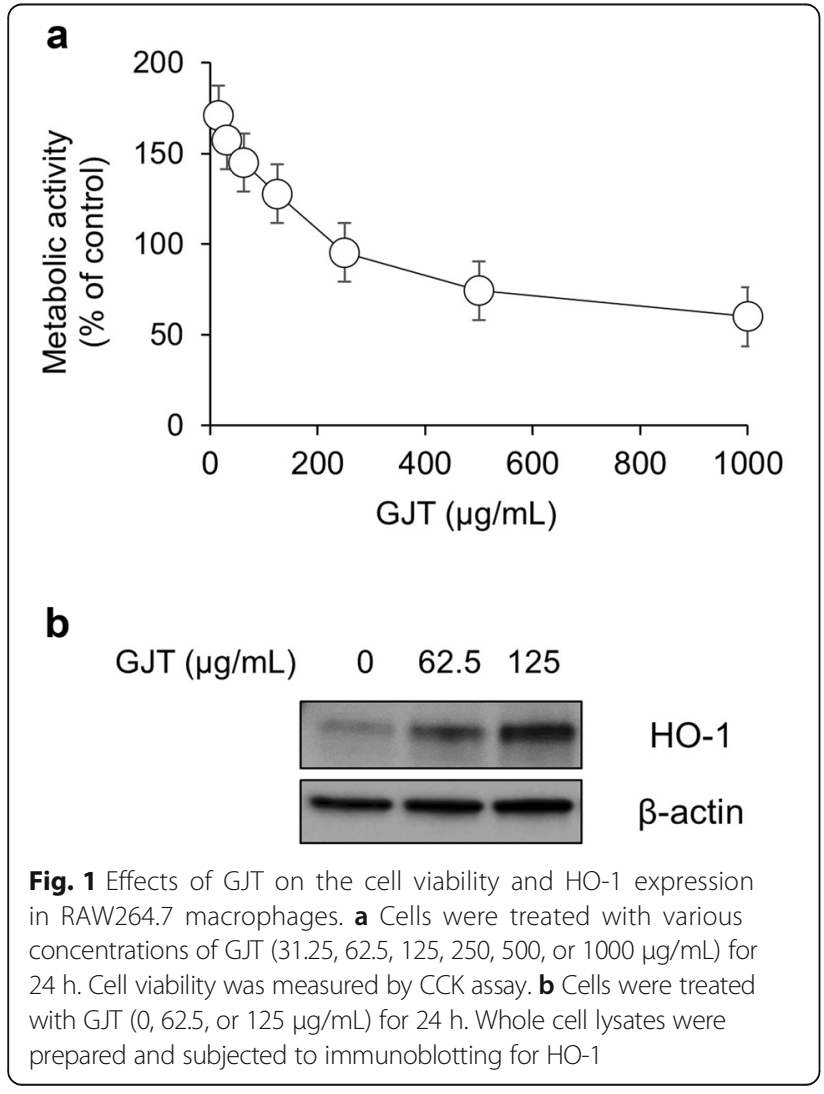

GJT suppresses TNF- $a$ and IL- 6 secretion in LPSstimulated RAW264.7 cells

To examine whether GJT can influence secretion of proinflammatory cytokines TNF- $\alpha$ and IL-6, GJT extracts were added into LPS-stimulated RAW 264.7 cells. LPS significantly enhanced the amounts of secreted TNF- $\alpha$ by $8694.92 \pm 560.8 \mathrm{pg} / \mathrm{mL}$ compared with untreated control $(6.06 \pm 6.06 \mathrm{pg} / \mathrm{mL})$. In contrast, GJT significantly reduced TNF- $\alpha$ production in a dose-dependent manner $(6318.46 \pm 6.06 \mathrm{pg} / \mathrm{mL}$ at $62.5 \mu \mathrm{g} / \mathrm{mL}$ and $4790.84 \pm$ $281.17 \mathrm{pg} / \mathrm{mL}$ at $125 \mu \mathrm{g} / \mathrm{mL}$ ) compared with LPSstimulated control (Fig. 2a, left panel). In RAW 264.7 cells, LPS stimulation also increased secretion of IL- 6 by $133.50 \pm 6.08 \mathrm{ng} / \mathrm{mL}$. Treatment with GJT at $125 \mu \mathrm{g} / \mathrm{mL}$ significantly suppressed IL-6 production by $103.10 \mathrm{ng} / \mathrm{mL}$ compared with LPS stimulation (Fig. 2b, left panel). Treatment with GJT alone weakly increased levels of TNF- $\alpha$ and IL-6 compared with untreated control (Fig. 2a and b, right panels).

\section{GJT inhibits $\mathrm{PGE}_{2}$ production and COX-2 expression in LPS-stimulated RAW264.7 cells}

$\mathrm{PGE}_{2}$ is a key inflammatory mediator synthesized by COX-2 [6]. To support the observed inhibitory effect of GJT on inflammatory response, a $\mathrm{PGE}_{2}$ assay was conducted using LPS-stimulated RAW 264.7 cells with or without GJT extract. As shown in Fig. 3a (left panel), LPS stimulation significantly increased production of $\mathrm{PGE}_{2}$ by $15149.2 \pm 768.01 \mathrm{pg} / \mathrm{mL}$ compared with untreated control $(552.16 \pm 58.82 \mathrm{pg} / \mathrm{mL})$. By contrast, LPS-stimulated PGE $_{2}$ increase was significantly inhibited by GJT at 62.5 and $125 \mu \mathrm{g} / \mathrm{mL}$ by $12032.07 \pm 423.16$ and $9410.4 \pm 315.41 \mathrm{pg} / \mathrm{mL}$, respectively. Treatment with GJT alone showed a marginal increase in the production of $\mathrm{PGE}_{2}$ compared with untreated cells (Fig. 3a, right panel). Indomethacin used as a positive control also significantly decreased the level of $\mathrm{PGE}_{2}$ production by LPS-treated RAW 264.7 cells. Treatment with GJT extract consistently suppressed COX-2, but not COX1, mRNA expression induced by LPS stimulation (Fig. 3b), indicating the specificity of GJT as a COX-2 inhibitor.

\section{GJT abrogates ERK phosphorylation in LPS-stimulated RAW264.7 cells}

MAPK signaling pathways are considered as molecular targets for anti-inflammatory therapy [17]. Therefore, we investigated the effect of GJT on the LPS-mediated phosphorylation of MAPK family members JNK, ERK, and p38 MAPK in RAW 264.7 cells. The cells were pretreated with GJT $(0,62.5$, or $125 \mu \mathrm{g} / \mathrm{mL})$ for $4 \mathrm{~h}$ and stimulated with LPS for 15 min. As shown in Fig. 4, LPS stimulation markedly increased levels of phosphorylated p38 MAPK, ERK, and JNK compared with untreated 
a

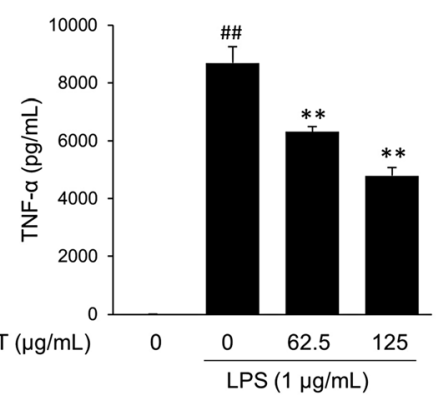

b

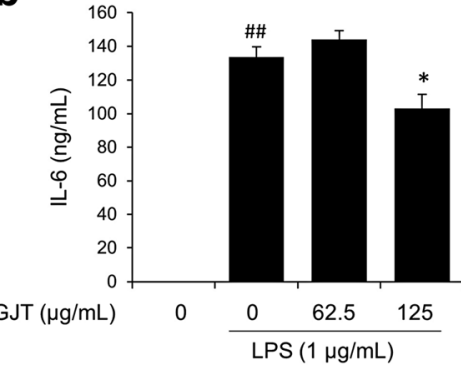

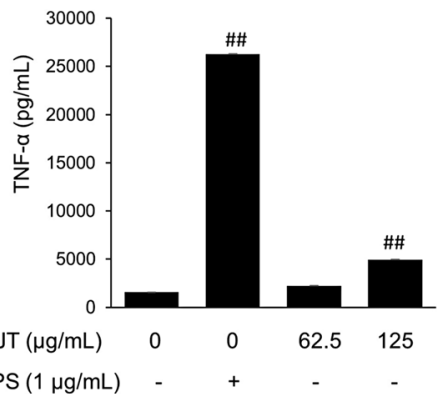

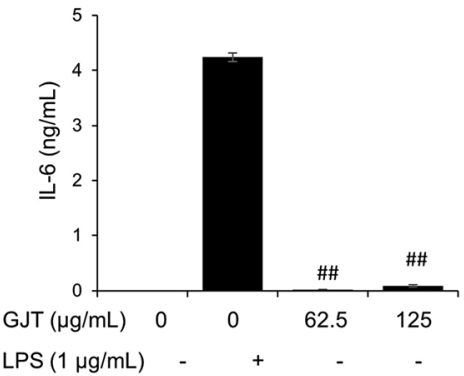

Fig. 2 Effects of GJT on production of TNF-a and IL-6 in LPS-stimulated RAW264.7 macrophages. Cells were pretreated with GJT (0, 62.5, or $125 \mu \mathrm{g} / \mathrm{mL})$ for $4 \mathrm{~h}$ and then treated with LPS $(1 \mu \mathrm{g} / \mathrm{mL})$ for $20 \mathrm{~h}$. Supernatants were collected and subjected to ELISAs for a TNF- $a$ and $\mathbf{b} \mathrm{IL}-6$. Bar graphs represent the mean \pm SEM from three independent experiments. ${ }^{\# \#} P<0.01$ vs vehicle control cells, and ${ }^{*} P<0.05$ and ${ }^{* *} P<0.01$ vs LPStreated cells. c; untreated control
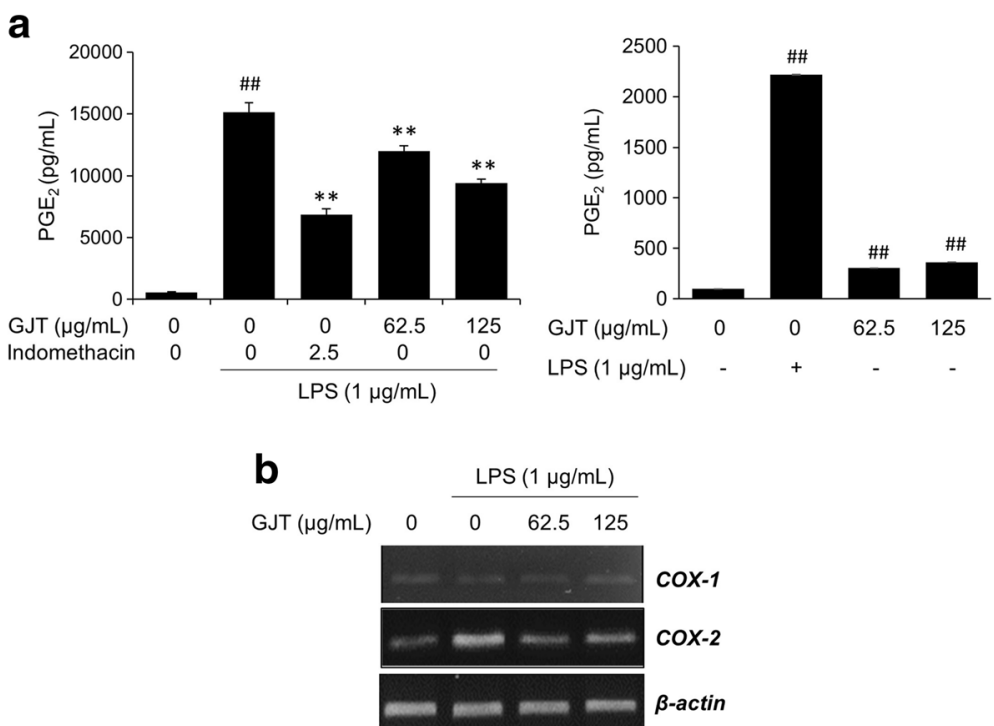

Fig. 3 Effects of GJT on PGE 2 production and COX-2 expression in LPS-stimulated RAW264.7 macrophages. Cells were pretreated with GJT (0, 62.5, or $125 \mu \mathrm{g} / \mathrm{mL})$ for $4 \mathrm{~h}$ and then treated with LPS $(1 \mu \mathrm{g} / \mathrm{mL})$ for $20 \mathrm{~h}$. a Supernatants were collected and subjected to ELISAs for PGE . b Total RNA was isolated from the cell pellets and subjected to RT-PCR for detecting COX-1 and COX-2 mRNA expression. Levels of COX-1 and COX-2 were adjusted by $\beta$-actin expression. Bar graphs represent the mean \pm SEM from three independent experiments. ${ }^{\# \#} P<0.01$ vs vehicle control cells, and ${ }^{* *} P<0.01$ vs LPS-treated cells. c; untreated control 


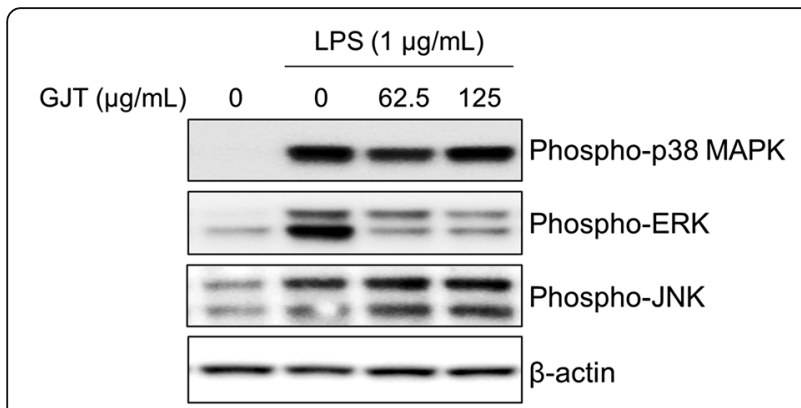

Fig. 4 Effects of GJT on activation of the MAPKs in LPS-stimulated RAW264.7 macrophages. Cells were pretreated with GJT $(0,62.5$, or $125 \mu \mathrm{g} / \mathrm{mL})$ for $2 \mathrm{~h}$ and the treated with LPS $(1 \mu \mathrm{g} / \mathrm{mL})$ for $15 \mathrm{~min}$. Cell lysates were prepared and subjected to immunoblotting for detecting the phosphorylation of P38 MAPK, ERK, and JNK. c; untreated control

cells. By contrast, treatment with GJT at 62.5 and $125 \mu \mathrm{g} / \mathrm{mL}$ reduced LPS-induced phosphorylation of ERK. However, GJT had no significant effect on JNK and p38 MAPK phosphorylation in LPS-treated RAW 264.7 cells.

\section{GJT inhibits NF-KB activation in LPS-stimulated RAW264.7}

cells

NF- $\mathrm{kB}$ signaling is involved in the development of inflammatory disorders [18]. We observed that GJT extract at $125 \mu \mathrm{g} / \mathrm{mL}$ markedly blocked LPS-stimulated nuclear expression of NF-kB p65 (Fig. 5a). Consistently, immunocytochemistry showed that LPS stimulation induced translocalization of NF- $\mathrm{kB}$ p 65 from the cytosol into the nucleus whereas with GJT extract blocked the LPS-mediated nuclear translocalization of NF- $\mathrm{kB}$ p65 in RAW 264.7 cells (Fig. 5b).

\section{HPLC analysis of GJT}

All calibration curves were obtained by plotting peak areas versus the concentration of standard solutions in seven different concentration ranges. The calibration curves of the 12 components showed good linearity with correlation coefficients $\left(r^{2}\right) \geq 0.9997$. The limit of detection (LOD) and quantitation (LOQ) were 9.15$258.61 \mathrm{ng} / \mathrm{mL}$ and $27.33-783.66 \mathrm{ng} / \mathrm{mL}$, respectively. These results are summarized in Table 2. Using optimized chromatography conditions, three-dimensional

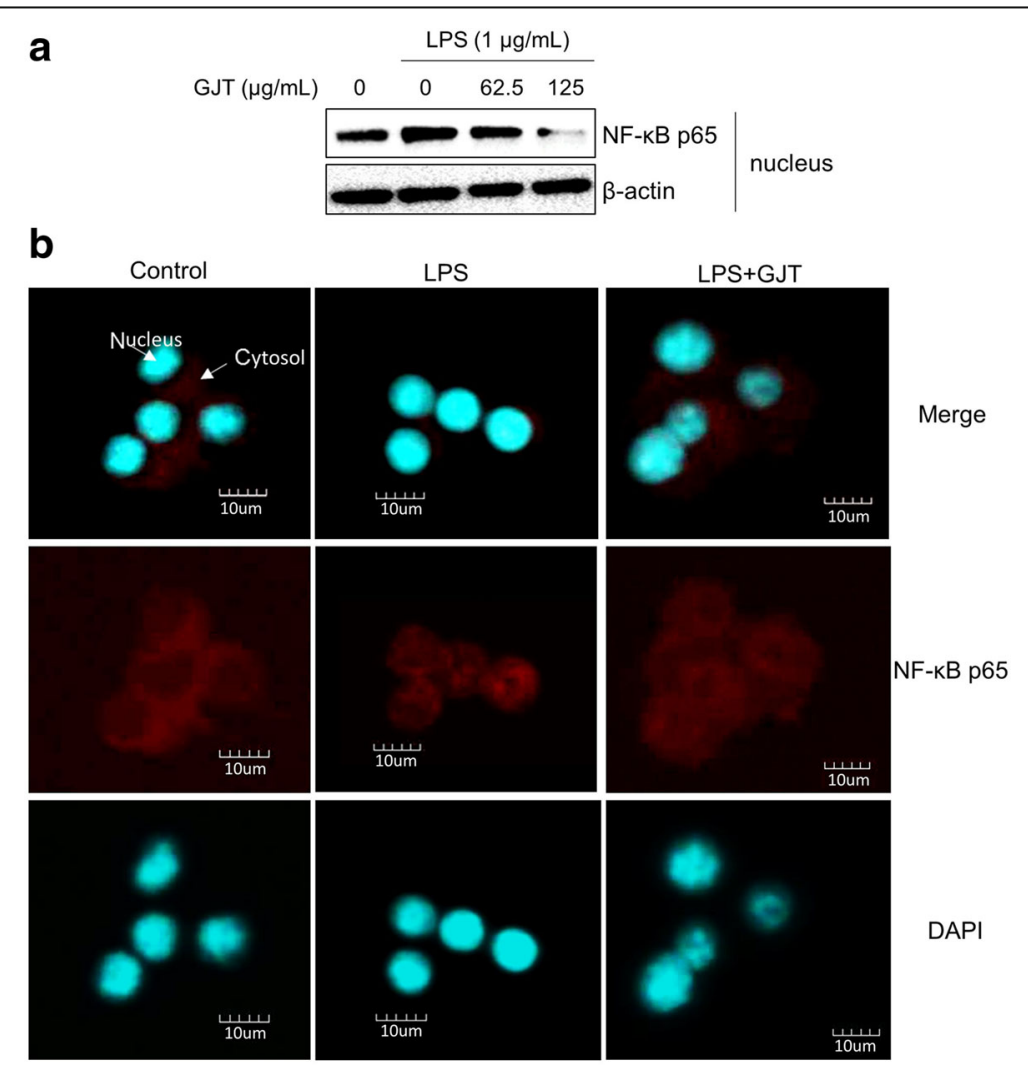

Fig. 5 Effects of GJT on activation of NF-KB in LPS-stimulated RAW264.7 macrophages. a Cells were pretreated with GJT (0, 62.5, or 125 $\mu \mathrm{g} / \mathrm{mL})$ for $2 \mathrm{~h}$ and the treated with LPS $(1 \mathrm{\mu g} / \mathrm{mL})$ for $1 \mathrm{~h}$. Nuclear extracts were prepared and subjected to immunoblotting for NF-KB p65. b Cells were pretreated with or without GJT (125 $\mu \mathrm{g} / \mathrm{mL})$ for $2 \mathrm{~h}$ and the treated with LPS $(1 \mu \mathrm{g} / \mathrm{mL})$ for $1 \mathrm{~h}$. The cells were fixed in $4 \%$ (v/v) methanol free formaldehyde solution ( $\mathrm{pH}$ 7.4), stained with anti-NF-KB p65 (Texas Red). The immunostained cells were then mounted with medium containing DAPI and visualized under an Olympus FLUOVIEW FV10i confocal microscopy. c; untreated control 
chromatograms were obtained using HPLC-PDA detector and 12 compounds were eluted within $35 \mathrm{~min}$ (Fig. 6). The amounts of the 12 marker compounds were $0.16-40.29 \mathrm{mg} / \mathrm{g}$ and are shown in Table 3.

\section{Discussion}

Inflammation is part of the abnormal body response caused by physical, biological or chemical stimuli [3]. Nonsteroidal anti-inflammatory drugs (NSAIDs) are the most commonly used for treating inflammatory disorders. NSAIDs target the COX enzyme and include aspirin, ibuprofen, and naproxen. However, long-term use of NSAIDs can trigger severe side effects such as gastric erosion, renal damage, myocardial infarction and asthma exacerbation [19]. Thus, novel therapeutic drugs with higher efficacy and fewer side effects are necessary for the treatment of inflammatory disorders.

In the present study, we investigated whether a traditional herbal formula GJT has anti-inflammatory effects in RAW 264.7 murine macrophages. An inflammatory reaction was induced by LPS treatment according to the previous reports [20,21]. Consistently, our data revealed that LPS stimulation significantly increased levels of proinflammatory cytokines TNF- $\alpha$ and IL-6 in RAW 264. 7 cells. By contrast, GJT treatment inhibited LPS-induced secretion of TNF- $\alpha$ and IL- 6 without cytotoxicity. Antiinflammatory activity of GJT extract was further confirmed by analyzing the expressions of $\mathrm{PGE}_{2}$ and COX2. Inflammatory stimulators such as LPS can induce COX-2 expression that produces $\mathrm{PGE}_{2}$, a key inflammatory mediator [22]. In the present study, GJT extract significantly decreased LPS-stimulated $\mathrm{PGE}_{2}$ production and COX-2 mRNA expression in RAW 264.7 cells. HO1 overexpression inhibits proinflammatory cytokine production and suppresses proinflammatory enzymes [23]. Thus, we investigated whether GJT influence on the expression of HO-1. We found that GJT treatment enhanced protein expression of HO-1 in RAW 264.7 macrophages. However, dose-dependency of HO-1 expression by GJT was consistent with the production of TNF- $\alpha$, but not IL- 6 and PGE 2 . Further experiments will be required to determine the relationship of antiinflammatory and antioxidant activities of GJP in macrophages.

Molecular regulation of inflammatory responses is closely associated with several signaling pathways such as those of MAPK and NF- $\kappa B$. Macrophage stimulation with LPS induces phosphorylation of MAPK family proteins ERK1/2, JNK, and p38 MAPK [24]. We observed that LPS stimulation clearly increased levels of phosphorylated ERK1/2, JNK, and p38 MAPK in RAW 264.7 cells. By contrast, GJT extract suppressed phosphorylation of ERK1/2, but not JNK or p38 MAPK, in LPSstimulated cells, indicating the importance of ERK $1 / 2$ to the anti-inflammatory regulation of GJT extract. However, we cannot exclude the possible inhibition of NF- $\mathrm{kB}$ activation. Indeed, our immunoblotting and immunofluorescence staining data showed that GJT treatment clearly reduced the nuclear level of NF-kB p65. Overall, GJT potentially controls inflammatory markers by blocking ERK and NF- $\mathrm{KB}$ signaling pathways in macrophages.

As mentioned above, GJT consists of 5 different herbal medicines Cinnamomum cassia, Paeonia lactiflora, Glycyrrhiza uralensis, Zingiber officinale, and Zizyphus jujube with certain ratio based on 'Sang han lon'. Of interest, it has been reported anti-inflammatory effects of individual herbs of GJT [25-29]. In addition, the major components of the 5 medicinal herbs are known as

Table 2 Regression data, linear range, correlation coefficient, LOD and LOQ for marker compounds ( $n=3$ ) of GJT extract

\begin{tabular}{|c|c|c|c|c|c|}
\hline Compound & Linear range $(\mu \mathrm{g} / \mathrm{mL})$ & Regression equation $^{a}$ & Correlation coefficient $\left(r^{2}\right)$ & $\mathrm{LOD}^{\mathrm{b}}(\mathrm{ng} / \mathrm{mL})$ & $\mathrm{LOQ}^{\mathrm{c}}(\mathrm{ng} / \mathrm{mL})$ \\
\hline Gallic acid & $1.56-100.00$ & $y=41971.92 x+834.16$ & 1.0000 & 45.36 & 137.44 \\
\hline Albiflorin & $1.56-100.00$ & $y=11838.31 x+1993.39$ & 0.9999 & 197.61 & 598.81 \\
\hline Paeoniflorin & $1.56-100.00$ & $y=14000.91 x-3078.78$ & 1.0000 & 167.08 & 506.32 \\
\hline Liquiritin apioside & $1.56-100.00$ & $y=16282.26 x+2703.99$ & 0.9999 & 107.59 & 326.03 \\
\hline Liquiritin & $1.56-100.00$ & $y=18152.25 x+2757.62$ & 0.9999 & 96.51 & 292.45 \\
\hline Benzoic acid & $1.56-100.00$ & $y=45334.92 x+22784.19$ & 0.9997 & 51.60 & 156.37 \\
\hline Coumarin & $1.56-100.00$ & $y=49833.92 x+8656.87$ & 0.9999 & 35.15 & 106.53 \\
\hline Benzoylpaeoniflorin & $0.31-20.00$ & $y=28757.81 x+814.63$ & 0.9999 & 81.35 & 246.50 \\
\hline Cinnamic acid & $0.31-20.00$ & $y=61499.33 x+3384.72$ & 1.0000 & 28.49 & 86.32 \\
\hline Cinnamaldehyde & $1.56-100.00$ & $y=131035.51 x+59890.08$ & 0.9997 & 9.15 & 27.73 \\
\hline Glycyrrhizin & $1.56-100.00$ & $y=8787.34 x+4482.00$ & 1.0000 & 40.39 & 122.40 \\
\hline 6-Gingerol & $0.31-20.00$ & $y=5693.60 x+109.67$ & 1.0000 & 258.61 & 783.66 \\
\hline
\end{tabular}

a: peak area (mAU) of compounds; $x$ : concentration $(\mu \mathrm{g} / \mathrm{mL})$ of compounds

${ }^{b} \mathrm{LOD}=3.3 \times \mathrm{SD} / \mathrm{S}$. (SD is the standard deviation of the blanks and $S$ is the slope of the calibration curve)

${ }^{\mathrm{C}} \mathrm{LOQ}=10 \times \mathrm{SD} / \mathrm{S}$ 


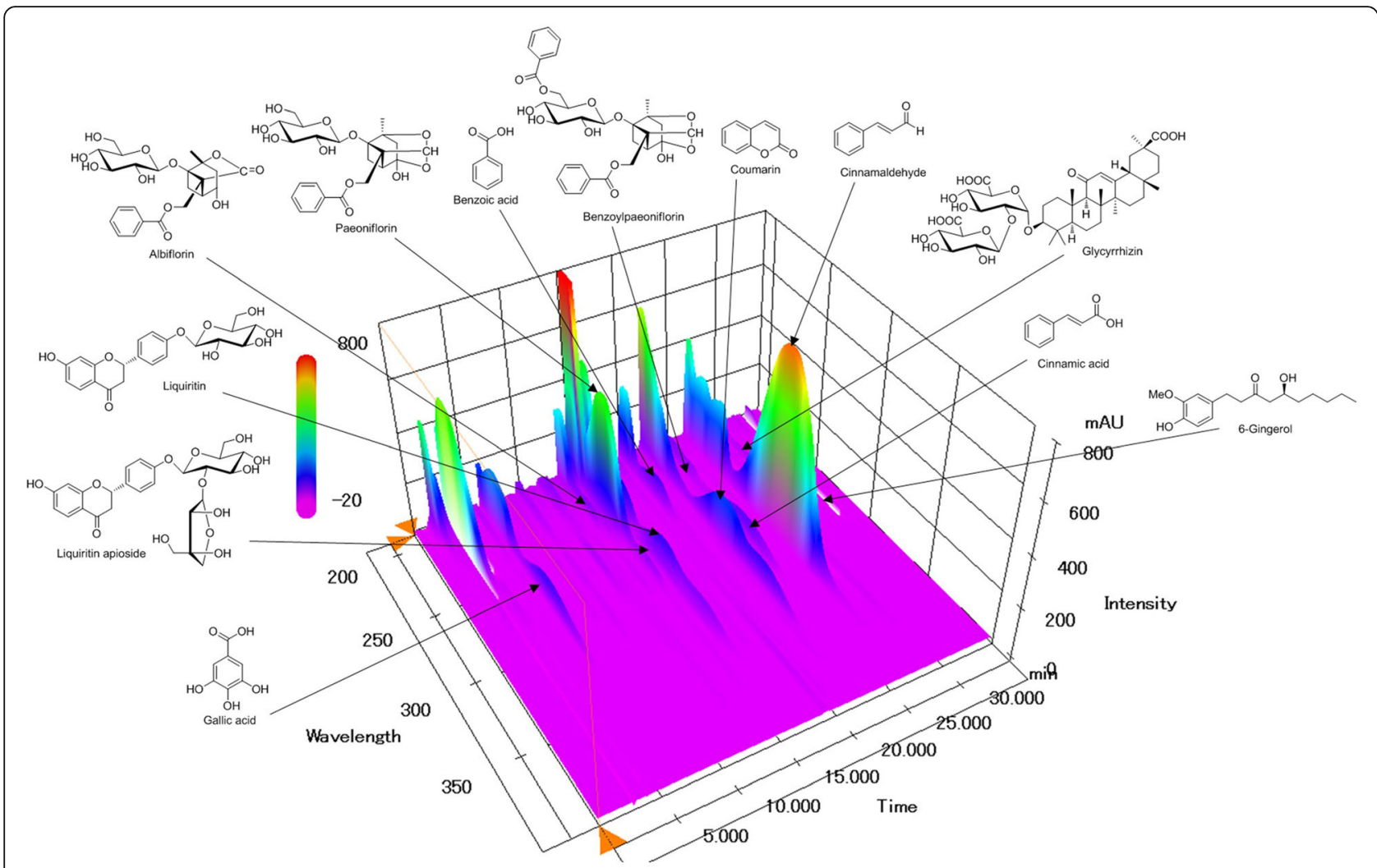

Fig. 6 Three-dimensional chromatogram of GJT by HPLC-PDA

follows: coumarins (e.g. coumarin) and phenylpropanoids (e.g. cinnamic acid and cinnamaldehyde) from C. cassia $[30,31]$, monoterpenoids (e.g. albiflorin and paeoniflorin) from $P$. lactiflora, triterpene saponin (e.g. glycyrrhizin) and flavonoids (e.g. liquiritin and liquiritigenin), from $G$. uralensis [32], phenols (e.g. 6-, 8-, and 10-gingerol) from Z. officinale [33], and flavonoids (e.g. spinosin and 6"'-

Table 3 Contents of 12 compounds in the GJT by HPLC $(n=3)$

\begin{tabular}{lcccl}
\hline Compound & Mean $(\mathrm{mg} / \mathrm{g})$ & $\mathrm{SD} \times 10^{-1}$ & $\mathrm{RSD}(\%)$ & Source $^{\mathrm{a}}$ \\
\hline Gallic acid & 3.18 & 0.89 & 2.79 & $\mathrm{PR}$ \\
Albiflorin & 2.78 & 0.42 & 1.52 & $\mathrm{PR}$ \\
Paeoniflorin & 40.29 & 1.17 & 0.29 & $\mathrm{PR}$ \\
Liquiritin apioside & 4.94 & 0.80 & 1.61 & $\mathrm{GRR}$ \\
Liquiritin & 6.61 & 0.26 & 0.39 & $\mathrm{GRR}$ \\
Benzoic acid & 2.60 & 0.30 & 1.14 & $\mathrm{PR}$ \\
Coumarin & 5.68 & 0.11 & 0.19 & $\mathrm{CR}$ \\
Benzoylpaeoniflorin & 0.72 & 0.19 & 2.58 & $\mathrm{PR}$ \\
Cinnamic acid & 1.76 & 0.09 & 0.54 & $\mathrm{CR}$ \\
Cinnamaldehyde & 9.32 & 0.08 & 0.08 & $\mathrm{CR}$ \\
Glycyrrhizin & 9.91 & 0.71 & 0.71 & $\mathrm{GRR}$ \\
6-Gingerol & 0.16 & 0.04 & 2.41 & ZRC \\
\hline
\end{tabular}

a $P R$ Paeoniae Radix, GRR Glycyrrhizae Radix et Rhizoma, $C R$ Cinnamomi Ramulus, ZRC Zingiberis Rhizoma Crudus feruloylspinosin) from $Z$. jujube [34]. Among those constituents, we analyzed 12 compounds, such as galiic acid, albiflorin, paeoniflorin, liquiritin apioside, liquiritin, benzoic acid, coumarin, cinnamic acid, benzoyl paeoniflorin, cinnamaldehyde, glycyrrhizin, and 6-gingerol using HPLC-PDA. An optimized HPLC-PDA method was applied for quantitative analysis of these 12 compounds in the GJT water extract. Consequently, paeoniflorin (40.29 $\pm 0.12 \mathrm{mg} / \mathrm{g}$ ), which is marker compound of P. lactiflora, was detected as the most abundant component in GJT extract. Anti-inflammatory activities of several GJT component compounds have been reported, including gallic acid [35], paeoniflorin [36], benzoic acid [37], cinnamaldehyde [38], glycyrrhizin [39], and 6-gingerol [40]. Further studies, including in animal models, will be required to elucidate the precise pharmacological mechanisms of the active compounds from GJT extract and their pharmacokinetics/ pharmacodynamics.

\section{Conclusions}

Our results show that an water extract of the traditional herbal formula GJT inhibits LPS-stimulated production of proinflammatory cytokines TNF- $\alpha$ and IL-6, and proinflammatory mediator $\mathrm{PGE}_{2}$ in macrophages. Antiinflammatory activity of GJT is associated with enhancement of $\mathrm{HO}-1$ expression and regulated via targeting 
ERK and NF-кB pathways. These findings suggest the prophylactic effects if GJT decoction on inflammatory diseases. Further studies will be considered in the near future to determine the effects of GJT for treating inflammatory diseases by the concurrent or post LPS treatment with GJT decoction.

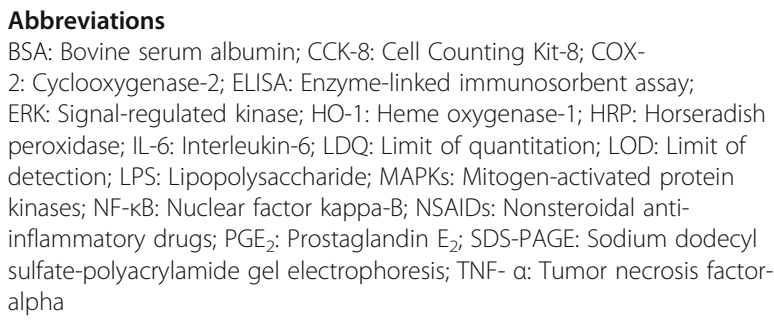

\section{Acknowledgement}

This research was supported by a grant for "Construction of Scientific Evidence for Herbal Medicine Formulas (K16251)", from the Korea Institute of Oriental Medicine (KIOM).

\section{Funding}

This research was supported by a grant for "Construction of Scientific Evidence for Herbal Medicine Formulas (K16251)", from the Korea Institute of Oriental Medicine (KIOM).

\section{Availability of data and materials}

The datasets supporting the conclusions of this article are included within the article.

\section{Authors' contributions}

SRY, YK and SJJ participated in the design of the study data analyses and manuscript preparation. MYL, OSK, CSS and HKS performed experiments. All authors read and approved the final manuscript.

\section{Competing interests}

The authors declare that they have no competing interests.

\section{Consent for publication}

Not applicable.

\section{Ethics approval and consent to participate}

Not applicable.

\begin{abstract}
Author details
'K-herb Research Center, Korea Institute of Oriental Medicine, Daejeon 34054, Republic of Korea. ${ }^{2} \mathrm{KM}$ Convergence Research Division, Korea Institute of Oriental Medicine, 1672 Yuseong-daero, Yuseong-gu, Daejeon 34054, Republic of Korea. ${ }^{3}$ Mucosal Immunology Laboratory, Department of Convergence Medicine, University of Ulsan College of Medicine/Asan Medical Center, Seoul, Republic of Korea. ${ }^{4}$ Korean Medicine Life Science, University of Science and Technology, Daejeon, Republic of Korea.
\end{abstract}

Received: 9 September 2015 Accepted: 23 September 2016

Published online: 12 October 2016

\section{References}

1. Philip M, Rowley DA, Schreiber $H$. Inflammation as a tumor promoter in cancer induction. Semin Cancer Biol. 2004;14(6):433-9.

2. Coussens LM, Werb Z. Inflammation and cancer. Nature. 2002;420(6917):860-7.

3. Fujiwara N, Kobayashi K. Macrophages in inflammation. Curr Drug Targets Inflamm Allergy. 2005;4(3):281-6.

4. Esposito E, Cuzzocrea S. TNF-alpha as a therapeutic target in inflammatory diseases, ischemia-reperfusion injury and trauma. Curr Med Chem. 2009;16(24):3152-67.

5. Tanaka T, Narazaki M, Kishimoto T. Therapeutic targeting of the interleukin-6 receptor. Annu Rev Pharmacol Toxicol. 2012;52:199-219.
6. Kang YJ, Mbonye UR, DeLong CJ, Wada M, Smith WL. Regulation of intracellular cyclooxygenase levels by gene transcription and protein degradation. Prog Lipid Res. 2007;46(2):108-25.

7. Saklatvala J, Dean J, Clark A. Control of the expression of inflammatory response genes. Biochem Soc Symp. 2003;70:95-106.

8. Paine A, Eiz-Vesper B, Blasczyk R, Immenschuh S. Signaling to heme oxygenase-1 and its anti-inflammatory therapeutic potential. Biochem Pharmacol. 2010;80(12):1895-903.

9. Naito Y, Takagi T, Higashimura Y. Heme oxygenase-1 and anti-inflammatory M2 macrophages. Arch Biochem Biophys. 2014;564:83-8.

10. Morse D, Pischke SE, Zhou Z, Davis RJ, Flavell RA, Loop T, Otterbein SL, Otterbein LE, Choi AM. Suppression of inflammatory cytokine production by carbon monoxide involves the JNK pathway and AP-1. J Biol Chem. 2003;278(39):36993-8.

11. Lee TS, Tsai HL, Chau LY. Induction of heme oxygenase-1 expression in murine macrophages is essential for the anti-inflammatory effect of low dose 15deoxy-Delta 12,14-prostaglandin J2. J Biol Chem. 2003;278(21):19325-30.

12. Suh GY, Jin Y, Yi AK, Wang XM, Choi AM. CCAAT/enhancer-binding protein mediates carbon monoxide-induced suppression of cyclooxygenase-2. Am J Respir Cell Mol Biol. 2006;35(2):220-6.

13. Hur J. Donguibogam. Republic of Korea: Namsandang. 2004. p. 380.

14. Du XL, Sui F, Huo HR, Zhang HW, Luo K, Li LF, Guo SY, Jiang TL. Reciprocal effects of Guizhi decoction to the Guizhi decoction syndrome by toll-like receptor mRNA expression and cytokines secretion. Chin J Integr Med. 2013;19(11):826-35.

15. Li X, Jiang YH, Jiang P, Yang JL, Ma DF, Yang CH. Effect of Guizhi Decoction ([symbols; see text]) on heart rate variability and regulation of cardiac autonomic nervous imbalance in diabetes mellitus rats. Chin J Integr Med. 2014;20(7):524-33.

16. Motoo Y, Su SB, Xie MJ, Mouri H, Taga H, Sawabu N. Effect of herbal medicine keishi-to (TJ-45) and its components on rat pancreatic acinar cell injuries in vivo and in vitro. Pancreatology. 2001;1(2):102-9.

17. Kaminska B. MAPK signalling pathways as molecular targets for antiinflammatory therapy-from molecular mechanisms to therapeutic benefits. Biochim Biophys Acta. 2005;1754(1-2):253-62

18. Liu SF, Malik AB. NF-kappa B activation as a pathological mechanism of septic shock and inflammation. Am J Physiol Lung Cell Mol Physiol. 2006:290(4):L622-45.

19. Trelle $S$, Reichenbach $S$, Wandel S, Hildebrand P, Tschannen B, Villiger PM, Egger $M$, Juni P. Cardiovascular safety of non-steroidal anti-inflammatory drugs: network meta-analysis. BMJ. 2011;342:C7086.

20. Keyel PA, Heid ME, Salter RD. Macrophage responses to bacterial toxins: a balance between activation and suppression. Immunol Res. 2011;50(2-3):118-23.

21. Wright SD, Ramos RA, Tobias PS, Ulevitch RJ, Mathison JC. CD14, a receptor for complexes of lipopolysaccharide (LPS) and LPS binding protein. Science. 1990;249(4975):1431-3.

22. Mitchell JA, Larkin S, Williams TJ. Cyclooxygenase-2: regulation and relevance in inflammation. Biochem Pharmacol. 1995;50(10):1535-42.

23. Pae HO, Lee YC, Chung HT. Heme oxygenase-1 and carbon monoxide: emerging therapeutic targets in inflammation and allergy. Recent Pat Inflamm Allergy Drug Discov. 2008;2(3):159-65.

24. Choi CY, Park KR, Lee JH, Jeon YJ, Liu KH, Oh S, Kim DE, Yea SS. Isoeugenol suppression of inducible nitric oxide synthase expression is mediated by down-regulation of NF-kappaB, ERK1/2, and p38 kinase. Eur J Pharmacol. 2007:576(1-3):151-9.

25. Park HJ, Lee JS, Lee JD, Pyo JH, Kang JM, Choe HI, Kim SY, Shim BS, Lee JH, Lim S. The Anti-inflammatory Effect of Cinnamomi Ramulus. J Korean Oriental Med. 2005;26(2):140-51.

26. He DY, Dai SM. Anti-inflammatory and immunomodulatory effects of paeonia lactiflora pall., a traditional chinese herbal medicine. Front Pharmacol. 2011;2:10.

27. Yang $\mathrm{XL}$, Liu D, Bian K, Zhang DD. [Study on in vitro anti-inflammatory activity of total flavonoids from Glycyrrhizae Radix et Rhizoma and its ingredients]. Zhongguo Zhong Yao Za Zhi. 2013;38(1):99-104.

28. Jung HW, Yoon CH, Park KM, Han HS, Park YK. Hexane fraction of Zingiberis Rhizoma Crudus extract inhibits the production of nitric oxide and proinflammatory cytokines in LPS-stimulated BV2 microglial cells via the NFkappaB pathway. Food Chem Toxicol. 2009:47(6):1190-7.

29. Tamaoki J, Kondo M, Tagaya E, Takemura K, Konno K. Zizyphi fructus, a constituent of antiasthmatic herbal medicine, stimulates airway epithelial ciliary motility through nitric oxide generation. Exp Lung Res. 1996;22(3):255-66. 
30. Liang K, Cui S, Zhang Q, Bi K, Qian Z, Jia Y. [UPLC simultaneous determination of five active components in Cinnamomi Ramulus]. Zhongguo Zhong Yao Za Zhi. 2011;36(23):3298-301.

31. Wen KC, Huang CY, Liu FS. Determination of cinnamic acid and paeoniflorin in traditional Chinese medicinal preparations by high-performance liquid chromatography. J Chromatogr. 1992;593(1-2):191-9.

32. Zhang Q, Ye M. Chemical analysis of the Chinese herbal medicine Gan-Cao (licorice). J Chromatogr A. 2009;1216(11):1954-69.

33. Zick SM, Ruffin MT, Djuric Z, Normolle D, Brenner DE. Quantitation of 6-, 8and 10-Gingerols and 6-Shogaol in Human Plasma by High-Performance Liquid Chromatography with Electrochemical Detection. Int J Biomed Sci. 2010;6(3):233-40.

34. Niu C, Zhang J. Quantitative analysis and chromatographic fingerprinting of the semen zizyphi spinosae by ultra-high-performance liquid chromatography coupled with diode-array detector. J Sep Sci. 2011:34(21):2989-96.

35. Hsiang CY, Hseu YC, Chang YC, Kumar K, Ho TY, Yang HL. Toona sinensis and its major bioactive compound gallic acid inhibit LPS-induced inflammation in nuclear factor-kappaB transgenic mice as evaluated by in vivo bioluminescence imaging. Food Chem. 2013;136(2):426-34.

36. Liu H, Wang J, Wang J, Wang P, Xue Y. Paeoniflorin attenuates Abetainduced inflammation and chemotaxis of microglia in vitro and inhibits NFkappaB- and VEGF/Flt-1 signaling pathways. Brain Res. 2015;1618:149-58.

37. Nguyen PH, Zhao BT, Lee JH, Kim YH, Min BS, Woo MH. Isolation of benzoic and cinnamic acid derivatives from the grains of Sorghum bicolor and their inhibition of lipopolysaccharide-induced nitric oxide production in RAW 264.7 cells. Food Chem. 2015:168:512-9.

38. Muhammad JS, Zaidi SF, Shaharyar S, Refaat A, Usmanghani K, Saiki I, Sugiyama T. Anti-inflammatory effect of cinnamaldehyde in Helicobacter pylori induced gastric inflammation. Biol Pharm Bull. 2015;38(1):109-15

39. Lau A, Wang S, Liu W, Haig A, Zhang ZX, Jevnikar AM. Glycyrrhizic acid ameliorates HMGB1-mediated cell death and inflammation after renal ischemia reperfusion injury. Am J Nephrol. 2014;40(1):84-95.

40. Tzeng TF, Liou SS, Chang CJ, Liu IM. [6]-gingerol dampens hepatic steatosis and inflammation in experimental nonalcoholic steatohepatitis. Phytomedicine. 2015;22(4):452-61.

\section{Submit your next manuscript to BioMed Central and we will help you at every step:}

- We accept pre-submission inquiries

- Our selector tool helps you to find the most relevant journal

- We provide round the clock customer support

- Convenient online submission

- Thorough peer review

- Inclusion in PubMed and all major indexing services

- Maximum visibility for your research

Submit your manuscript at www.biomedcentral.com/submit

) Biomed Central 